\title{
Partners' Consensus About Joint Effort and COVID-19 Prevention Among Sexual Minority Men
}

\author{
Tyrel J. Starks ${ }^{1,2} \cdot$ Stephen C. Bosco ${ }^{2} \cdot$ Kendell M. Doyle $^{2} \cdot$ Tracey A. Revenson $^{1,2}$
}

Received: 24 October 2020 / Revised: 17 May 2021 / Accepted: 25 May 2021 / Published online: 21 June 2021

(c) The Author(s), under exclusive licence to Springer Science+Business Media, LLC, part of Springer Nature 2021

\begin{abstract}
The current study examined the relevance of relationship functioning to partners' agreement or consensus about joint effort surrounding COVID-19 prevention. Interdependence theory has been widely used to understand how relationship partners influence health behavior, including how sexual minority male (SMM) couples regulate HIV risk. Couples with better relationship functioning tend to be more successful at negotiating joint (shared) goals and subsequently accomplishing them. The study recruited 134 cis-male, SARS-CoV-2 negative adults in relationships with cis-male partners from phone-based social networking applications. Participants completed an online survey assessing relationship functioning (Perceived Relationship Components Questionnaire), COVID-19 prevention behaviors, and risk perceptions. Partners' consensus around joint COVID-19 prevention effort was assessed using an adapted version of the Preferences for Sexual Health Outcomes scale. Path analyses indicated that consensus for joint prevention effort predicted social distancing $(B=0.23 ; p=.001)$ and the number of other COVID-19 prevention behaviors engaged in $(B=0.17 ; p=.003)$ above and beyond perceived risk and relationship functioning. Relationship satisfaction predicted higher levels of consensus for joint COVID-19 prevention effort $(B=0.40$; $p=.029$ ). Findings suggest that the theoretical foundations of successful HIV prevention interventions that utilize joint goal formation may generalize to the prediction of COVID-19 prevention behavior and may be leveraged to mitigate the risk of SARS-CoV-2 infection among SMM in relationships. Interventions that overlook the potential for dyadic regulation of health behavior may miss opportunities to capitalize on shared coping resources and fail to address relational barriers to prevention.
\end{abstract}

Keywords COVID-19 $\cdot$ SARS-CoV-2 $\cdot$ Interdependence theory $\cdot$ Male couples $\cdot$ Perceived risk $\cdot$ Sexual orientation

\section{Introduction}

Given the constellation of biological, social, and structural risks faced by sexual minority men (SMM) in the U.S., there is reason to expect that they would be at greater risk of SARSCoV-2 infection and greater risk of severe COVID-19 complications if infected (Banerjee \& Nair, 2020; Sanchez et al., 2020). The prevalence of HIV is higher among SMM relative to the general population, enhancing vulnerability by weakening the immune system (Blosnich et al., 2016; Operario

Tyrel J. Starks

tstarks@ hunter.cuny.edu

1 Department of Psychology, Hunter College of the City University of New York (CUNY), New York, NY 10065, USA

2 Health Psychology and Clinical Science Doctoral Program, The Graduate Center of the City University of New York (CUNY), New York, NY, USA et al., 2015). SMM also have elevated rates of underlying health conditions that have been identified as risk factors for COVID-19, including heart disease, obesity, and asthma (Blosnich et al., 2016; Caceres et al., 2019; Curry et al., 2020; Veldhuis et al., 2019). SMM are less likely to have adequate health insurance (Charlton et al., 2018), have more limited access to healthcare services (Albuquerque et al., 2016; Romanelli \& Hudson, 2017), experience greater stigma from healthcare providers (Eaton et al., 2015; Sabin et al., 2015), and are less likely to utilize healthcare services when necessary (Nguyen et al., 2018).

Among COVID-19 behavioral prevention strategies, social distancing has received considerable attention. Social distancing is defined by the Centers for Disease Control and Prevention as "keeping a safe space between yourself and other people who are not from your household" (Centers for Disease Control and Prevention, 2020). Social distancing is a complex behavioral goal accomplished through a multifaceted set of behaviors (e.g., utilizing lower-contact methods 
for social interactions such as phone calls, limiting social activities, and utilizing low-contact transportation options). In addition to social distancing, a number of other health behaviors have been suggested to further reduce the spread of the SARS-CoV-2 virus, including wearing a face mask that covers both your nose and mouth, frequent hand washing, as well as cleaning and disinfecting surfaces (Centers for Disease Control and Prevention, 2020; Eikenberry et al., 2020; Lewnard \& Lo, 2020).

While social distancing and other behavioral prevention measures reduce SARS-CoV-2 infection risk (Courtemanche et al., 2020; Thunström et al., 2020), continuously enacting these precautions can be conceptualized as a psychosocial stressor (Brennan et al., 2020). SMM have been found to experience elevated levels of COVID-19-related stress. Among a sample of SMM, pandemic-related worry has been linked to sleep disturbance (Millar et al., 2020). SMM have reported challenges with meeting basic resource needs during the pandemic, such as purchasing food and paying rent due to loss of employment and limited access to sexual healthcare (i.e., HIV and STI testing, pre-exposure prophylaxis (PrEP) services, and HIV treatments; Banerjee \& Nair, 2020; Brennan et al., 2020; Sanchez et al., 2020). Social distancing has been shown to decrease quality of life, increase anxiety, and decrease connections to social support networks among SMM specifically (Brennan et al., 2020; Torres et al., 2020). Given the structural, social, and health-related inequalities faced by SMM, the additive effects associated with perceived COVID-19 risk and social distancing are of particular concern to their health and overall well-being.

Social distancing has been thought of largely as an individual behavior. However, for individuals in relationships, a level of coordination and joint action is required to enact social distancing and other behavioral strategies to prevent SARS-CoV-2 infection. Unless partners remain distanced from one another, one partner's failure to implement prevention practices presents a potential source of exposure for the other. These circumstances-where goal attainment requires coordination and cooperation between partners (termed joint control) - can be understood in the context of Interdependence Theory (Kelley \& Thibaut, 1978; Rusbult \& Van Lange, 2003; Rusbult et al., 1991). In situations requiring joint control, partners tend to be more successful at working together toward a shared goal when they have higher quality relationship functioning. Rusbult's Investment Model (Rusbult, 1980; Rusbult et al., 1998)—an extension of Interdependence Theory-posits that individuals who are more satisfied with, invested in, and committed to their relationships are more likely to consider the consequences of their actions for their partner and their relationship rather than primarily focusing on personal gains or costs (termed a transformation of motivation). This subsequently promotes adaptive or constructive responses such as problem-solving or providing emotional support (sometimes referred to as accommodation) in response to disagreements or inconsiderate behavior (Rusbult \& Van Lange, 2003; Rusbult et al., 1983, 1991). Consistent with the overall premise of Interdependence Theory, research with SMM, specifically, has illustrated associations between dimensions of relationship functioning and a range of health outcomes, including condom use (Davidovich et al., 2006; Goldenberg et al., 2015; Mitchell \& Petroll, 2013; Starks et al., 2014), HIV medication adherence (Goldenberg et al., 2013; Johnson et al., 2012), pre- and post-exposure prophylaxis to prevent HIV (Bosco et al., 2021; Gamarel \& Golub, 2019; Starks et al., 2019b), and substance use (Starks et al., 2019d).

Theories of dyadic coping further suggest that couples who perceive health concerns as a significant threat or shared stressor are more likely to view them as joint goals and take a dyadic approach to manage those health concerns (Berg \& Upchurch, 2007; Bodenmann et al., 2011; Falconier \& Kuhn, 2019; Lewis et al., 2006). This suggests that threat perceptions may influence couples' willingness to engaging in joint coping around health behaviors. This work has largely been conducted among heterosexual couples coping with chronic illness (e.g., cancer, diabetes, mental illness; Falconier \& Kuhn, 2019).

Investigations of partnered individuals suggest that risk perception influences an individual's likelihood of engaging in risk reduction behaviors. For example, among a sample of partnered SMM, men who perceived themselves as being at low risk of HIV acquisition had lower odds of engaging in regular testing. In contrast, SMM who believed themselves to be at a higher risk of HIV acquisition were more likely to engage in regular testing (Stephenson et al., 2015a). Although investigations in this area are limited, there is some evidence to suggest that SMM couples who perceive HIV transmission as a significant health threat and perceive the management of HIV transmission risk as a shared responsibility are more likely to engage in risk reduction strategies (e.g., condom use, PrEP uptake; Gamarel et al., 2014; Starks et al., 2019b).

It has been suggested that HIV prevention strategies can be leveraged to prevent SARS-CoV-2 infection among SMM populations (Eaton \& Kalichman, 2020). Existing dyadic interventions to reduce HIV acquisition risk (Grabbe et al., 2012; Newcomb et al., 2017; Starks et al., 2019a, 2020b; Stephenson et al., 2017) or improve HIV-related health among men with an HIV-positive status (Wu et al., 2015) assume that establishing a shared HIV prevention goal is a mechanism of change. Dyadic interventions, such as Couples HIV Testing and Counseling, encourage couples to discuss their perceived risk, formulate shared goals to reduce risk, and identify behavioral strategies that can be implemented to achieve these mutually agreed upon goals (Grabbe et al., 2012). These dyadic interventions have been found to significantly reduce HIV transmission risk (Remien et al., 2005; 
Stephenson et al., 2011; Sullivan et al., 2014). It is plausible that successful joint coping with COVID-19 prevention - that is, shared efforts to prevent SARS-CoV-2 - arises through similar interdependent processes and is facilitated by relationship functioning and risk perception in a manner analogous to HIV prevention. Demonstrating the viability of this premise is a necessary prerequisite to the translation of these prevention interventions for use with COVID-19.

The current study tests a hypothesized model predicting the implementation of COVID-19 prevention strategies from partners' consensus about joint effort for COVID-19 prevention. Consistent with interdependence theory, we hypothesized that greater consensus about a joint effort to prevent COVID-19 would predict more social distancing and a greater number of other COVID-19 prevention behaviors. In addition, we hypothesized that joint COVID-19 prevention efforts would be positively associated with indicators of relationship functioning as well as perceptions of COVID19 risk. These hypotheses were evaluated in both bivariate analyses and a multivariable path model controlling for demographic factors in the prediction of endogenous variables. In the latter, we hypothesized that significant indirect effects linking relationship functioning and risk perceptions with social distancing and COVID-19 prevention behaviors through consensus about joint effort for COVID-19 prevention would be significant.

\section{Method}

\section{Participants}

Eligible participants were aged 18 or older, reported a residence in the U.S., and were able to respond to a survey in English. The current analyses were limited to cis-male participants who indicated that they had a cis-male primary relationship partner. Consistent with a focus on COVID-19 prevention, analyses were further limited to those participants who indicated that they had not had a COVID-19 diagnosis or symptoms consistent with COVID-19 at the time of survey completion.

\section{Procedure}

Data were collected as part of the PRIDE Endures project (Starks et al., 2020a) - a larger cross-sectional survey whose purpose was to examine the impact of COVID-19 on sexual and gender minority individuals active in online social networking and dating spaces. Participants were recruited through advertisements on mobile phone-based social networking and dating applications between May 6-15, 2020. Participants who responded to recruitment advertisements were directed to consent information. Those who agreed to participate responded to an online survey hosted by Qualtrics. Following survey completion, participants had the option to provide contact information to be entered into a random drawing for one of 20 Amazon gift certificates worth \$50. All procedures were approved by the appropriate Institutional Review Board.

\section{Measures}

Demographic variables Participants reported their age, race and ethnicity, HIV status, education, and annual income. Participants indicated whether or not they had a main partner and, if so, provided information about relationship length and partner's HIV status.

Relationship functioning was assessed using an abbreviated version of the Perceived Relationship Quality Component (PRQC) scale (Fletcher et al., 2000), a measure that has been used in studies of heterosexual couples (Muusses et al., 2015; Silva et al., 2017) as well as SMM couples (Cooper et al., 2017; Godfrey et al., 2021). The survey included four of the six PRCQ domains. Three were retained based on their prominence in Interdependence Theory (Agnew et al., 1998; Johnson \& Rusbult, 1989; Rusbult, 1983; Rusbult et al., 1998): Satisfaction (3-items, e.g., "How content are you with your relationship?"), Commitment (3-items, e.g., "How devoted are you to your relationship?"), Intimacy (3-items, e.g., "How intimate is your partner?"). The fourth, Passion (3-items, e.g., "How sexually intense is your relationship?"), assesses elements of sexual satisfaction particularly relevant to this sample, which was recruited in online dating and social networking spaces frequently used to locate sexual partners. Each domain has three items. Participants indicated the extent to which each of the 12 items describes their current partner or relationship using a Likert scale from 1 (Not at all) to 7 (Extremely). Possible scores for each subscale range from 3 to 21, with higher scores indicating greater relationship functioning. Reliability was strong for all four subscales (Cronbach's $\alpha=0.98,0.93,0.85$, and 0.93 , respectively).

Perceived COVID-19 risk was assessed using a modified version of the Perceived Risk for HIV scale (Napper et al., 2012), which has been used extensively in studies of SMM (Cohen et al., 2015; Fisher et al., 2018; Macapagal et al., 2018). This self-report measure was adapted to assess participants' perceived risk of COVID-19 by substituting "COVID19" in place of "HIV." The measure assessed three different aspects of the perceived risk of COVID-19: the cognitive assessments of risk (e.g., "There is a chance, no matter how small, I could get COVID-19"), the affective or intuitive assessments (e.g., "I worry about getting infected COVID19"), and the salience of risk (e.g., "Picturing myself getting COVID-19 is something I find:" Very hard to do to Very easy to do). The response format varied from four to six options; total scores were calculated by summing responses. Possible 
scores range from 10 to 56, with higher scores indicating greater perceived risk. Reliability was adequate $(\alpha=0.79)$.

Consensus about joint COVID-19 prevention effort was assessed using a modified version of the Preferences for Sexual Health Outcomes scale (Salazar et al., 2013), which has been used previously in studies of partnered SMM (Mitchell et al., 2019). Participants indicated the extent to which they and their partner were "on the same page" about whether a series of seven COVID-19-related prevention behaviors will benefit the couple (e.g., "Staying home will protect us from COVID-19"; "Limiting our number of sex partners will product us from COVID-19"; "Social distancing will protect us from COVID-19"). All 7-items were scored on a 5-point Likert-type scale ranging from 0 (We don't agree at all) to 4 (We completely agree). Possible scores range from 0 to 28 , with higher scores indicating greater consensus about joint COVID-19 prevention effort. Reliability for the scale was excellent (Cronbach's $\alpha=0.90$ ).

\section{COVID-19 Prevention Strategies}

Social distancing was assessed using a single item. Preceding the question, social distancing was defined as "the act of staying away from situations where you may be in close contact with others, such as social gatherings, work, school, sporting gatherings, restaurants, faith-based gatherings, and other public gatherings." After reading that statement, respondents were asked to indicate the extent to which they were socially distancing by choosing one of five response options: "None of the time-I am doing everything I normally do"; "Some of the time. I am limiting social interactions to family members who live in my community"; "Some of the time. I have reduced the amount of time I am in public spaces, social gathers or at work"; "Most of the time. I only leave my home to buy food and other essentials"; and "All of the time. I am staying at home nearly all the time." Responses to both options that began with "some of the time" were combined to create a 4-point ordinal scale ranging from 1 (None of the time) to 4 (All of the time).

Other COVID-19 prevention behaviors Participants indicated whether or not they engaged in each of four specific COVID-19 prevention behaviors (yes/no): remain six feet away from others; wear a face mask; wear latex or rubber gloves; and wash hands immediately upon returning home. These responses were counted to yield a score (0-4), indicating the number of COVID-19 prevention behaviors participants utilized. The use of a summed score was supported by confirmatory factor analysis, indicating that responses to these behavioral items were loaded on a single latent factor. Loadings for all four behaviors were statistically significant. Although the upper-bound of the 95\% confidence interval for the root mean square error of approximation (RMSEA) was higher than the common threshold for adequate fit
(Weston \& Gore, 2006), all other indices indicated good fit $\left(\chi^{2}(2)=2.618, p=.27\right.$; confirmatory fit index $=0.98$; standardized root mean square residual $=0.078 ; \mathrm{RMSEA}=0.048$, upper-bound of the $95 \%$ confidence interval $=0.185$ ).

\section{Data Analysis Plan}

Descriptive and bivariate statistics were calculated in SPSS version 25. Mplus (v. 8.2.; Muthén \& Muthén, 2018) was used to test the hypothesized path model. COVID-19 prevention strategies-social distancing and other COVID-19 prevention behaviors - were both specified as ordinal outcomes predicted by consensus for joint COVID-19 prevention effort, perceived COVID-19 risk, and relationship functioning. In addition, consensus for joint COVID-19 prevention effort was regressed on perceived COVID-19 risk and relationship functioning. The model included age, race and ethnicity, respondent HIV status, partner HIV status, and education as covariates in the prediction of all endogenous variables.

Although some subgroups of race and ethnicity were modest in size, no problems with model convergence were observed. We also tested models in which race and ethnicity were operationalized as a binary (majority White versus non-White) variable. The results of this model did not differ substantively from the results of a model that included the 4-category variable. We, therefore, report models that retain the 4-category race and ethnicity variable.

The significance of indirect paths was evaluated using the Model Test command in Mplus (v. 8.2.; Muthén \& Muthén, 2018). In these analyses, all tested indirect effects were comprised of two direct effects. For example, the indirect path from relationship satisfaction to social distancing through consensus for joint COVID-19 prevention effort was comprised of the direct path between consensus for joint COVID19 prevention effort and the direct path from relationship satisfaction to consensus for joint COVID-19 prevention effort. Using the model test command, the fit of a model in which the product of these two constituent paths is constrained to be zero is compared to one wherein the product is allowed to vary freely using a Wald $\chi^{2}$ test. A statistically significant test statistic indicates that the constraint diminishes model fit and provides support for the significance of the indirect path.

\section{Results}

Overall, 1001 participants viewed consent information and began the survey. Of these, $730(72.9 \%)$ responded to the main partner question, and of these 730 men, 193 (26.4\%) indicated they had a main partner. However, 62 (32.1\%) of the men with partners were excluded because they did not meet eligibility criteria: 12 identified as transgender or non-binary; 26 indicated their main partner was female or transgender, 
and 15 indicated they had COVID-19. This resulted in a final sample of 134 participants. Table 1 contains a detailed summary of the sample. Participants primarily identified as White (56.7\%) or Black (27.6\%), HIV-negative (74.6\%), and Gay $(88.1 \%)$. The average age was $43.1(\mathrm{SD}=13.4)$, and the average relationship length was 7.9 years $(\mathrm{SD}=8.3)$.

Table 2 contains bivariate associations among constructs of primary interest. Consistent with Interdependence Theory, consensus for joint COVID-19 prevention effort was positively and significantly associated with relationship satisfaction, commitment, and intimacy, though not with relationship passion. Also consistent with Interdependence Theory, consensus for joint COVID-19 prevention effort was positively associated with both the degree to which participants were social distancing (Kendall's $T=0.195$, $p<.05)$ and the number of prevention strategies endorsed (Kendall's $T=0.276, p<.01$ ).

Table 1 Sample characteristics

\begin{tabular}{|c|c|}
\hline & $\begin{array}{l}\text { Total } \\
n(\%)\end{array}$ \\
\hline$n$ & $134(100)$ \\
\hline \multicolumn{2}{|l|}{ Race and ethnicity } \\
\hline White/European & $76(56.7)$ \\
\hline Black/African American & $37(27.6)$ \\
\hline Latino & $14(10.4)$ \\
\hline Other & $7(5.2)$ \\
\hline \multicolumn{2}{|l|}{ Education } \\
\hline Less than 4-year college degree & $67(50.0)$ \\
\hline 4-year college degree or higher & $67(50.0)$ \\
\hline \multicolumn{2}{|l|}{ HIV status } \\
\hline Negative & $100(74.6)$ \\
\hline Positive & $34(25.4)$ \\
\hline \multicolumn{2}{|l|}{ Partner HIV status } \\
\hline Negative & $104(77.6)$ \\
\hline Positive & $30(22.4)$ \\
\hline \multicolumn{2}{|l|}{ Sexual identity } \\
\hline Gay & $118(88.1)$ \\
\hline Bisexual & $14(10.4)$ \\
\hline Other & $2(1.5)$ \\
\hline \multicolumn{2}{|l|}{ Social distancing } \\
\hline All of the time & $22(16.4)$ \\
\hline Most of the time & $63(47.0)$ \\
\hline Some of the time & $37(27.6)$ \\
\hline \multirow[t]{2}{*}{ None of the time } & $12(9.0)$ \\
\hline & $M(\mathrm{SD})$ \\
\hline Age (in years) & $43.1(13.4)$ \\
\hline Relationship duration (in months) & $7.9(8.3)$ \\
\hline \multicolumn{2}{|l|}{ Relationship functioning } \\
\hline Satisfaction & $15.2(4.6)$ \\
\hline Commitment & $16.8(4.3)$ \\
\hline Intimacy & $15.1(4.4)$ \\
\hline Passion & $11.7(5.7)$ \\
\hline Perceived COVID risk & $32.9(6.9)$ \\
\hline Joint COVID prevention effort & $22.7(5.0)$ \\
\hline Prevention behaviors & $2.8(1.0)$ \\
\hline
\end{tabular}




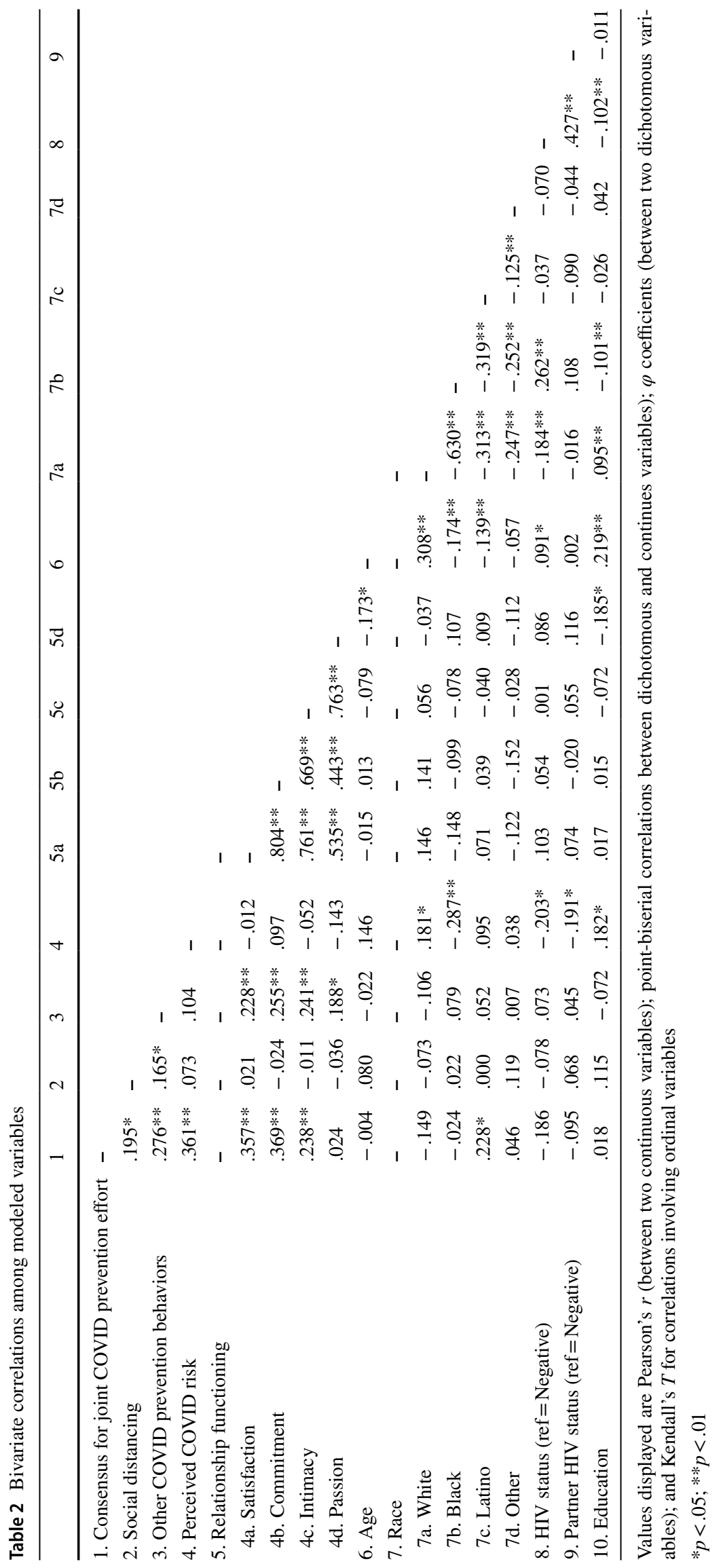




\section{Predictors of Social Distancing}

Figure 1 displays the results of the hypothesized path model predicting social distancing and other COVID-19 prevention behaviors. An omnibus test of model significance was conducted by comparing the log-likelihood value for the specified model to that of a baseline model wherein all the structural pathways were constrained to be zero using procedures specified by Satorra and Bentler (2001). This omnibus test was significant $\left(\chi^{2}(41)=107.80, p<.001\right)$.

Table 3 contains full results for all coefficients calculated. Consensus for joint COVID-19 prevention effort was significantly associated with level of social distancing $(B=0.23$; 95\% CI: 0.09-0.37; $\exp B=1.26 ; p=.001)$, but not with the relationship functioning subscales, perceived risk, or any demographic variables.

\section{Predictors of Other COVID-19 Prevention Behaviors}

Mirroring the results for social distancing, consensus for joint COVID-19 prevention effort was significantly associated with the number of COVID-19 prevention behaviors endorsed ( $B=0.17$; 95\% CI: 0.06-0.28; $\exp B=1.19$; $p=.003$ ). Relationship functioning subscales, perceived risk, and demographic characteristics were all non-significant.

\section{Predictors of Joint COVID Prevention Effort}

Of the relationship functioning subscales, only relationship satisfaction $(B=0.40 ; 95 \%$ CI: $0.04-0.77 ; \beta=.34 ; p=.029)$ was positively associated with consensus for joint COVID19 prevention effort, as was perceived COVID-19 risk $(B=0.28 ; 95 \%$ CI: $0.12-0.49 ; \beta=.36 ; p<.001)$. With respect to demographic characteristics, only race and ethnicity contributed significantly to the prediction of consensus for joint
COVID-19 prevention effort. Black participants $(B=2.77$; 95\% CI: $0.30-5.20 ; \beta=.22 ; p=.025)$; Latino participants $(B=4.63 ; 95 \%$ CI: $2.30-6.88 ; \beta=.26 ; p<.001)$; and participants in the "Other" category (American Indian or Alaskan Native, Asian, Native Hawaiian, or Other Pacific Islander; $B=3.44$; 95\% CI: $0.05-6.72 ; \beta=.14 ; p=.04)$ all had significantly higher scores on consensus for joint COVID-19 prevention effort scores compared to those men who identified as White.

\section{Testing Indirect Paths Involving Joint COVID-19 Prevention Effort}

The small number of significant direct effects constrained the number of indirect paths tested. Because constraining a product term to be zero when one of the values involved in that multiplication is not significantly different from zero will not yield a significant result, the pattern of direct effects suggested four possible indirect paths. As shown in Fig. 1, each of these four paths involved consensus for joint COVID-19 prevention effort as the intermediary variable, with two predicting social distancing behavior and two predicting other COVID-19 prevention behaviors.

Social distancing The path from perceived risk through consensus for joint COVID-19 prevention effort was statistically significant (Wald $\chi^{2}(1)=6.52 ; p=.01$ ). However, the path from relationship satisfaction through consensus for joint COVID-19 prevention effort was non-significant (Wald $\left.\chi^{2}(1)=3.02 ; p=.08\right)$.

Other COVID-19 prevention behaviors Mirroring the results above, the path from perceived risk through consensus for joint COVID-19 prevention effort was statistically significant (Wald $\chi^{2}(1)=5.51 ; p=.02$ ). The path from relationship satisfaction through joint COVID-19 prevention effort was at the cutoff for significance (Wald $\chi^{2}(1)=3.84 ; p=.05$ ).

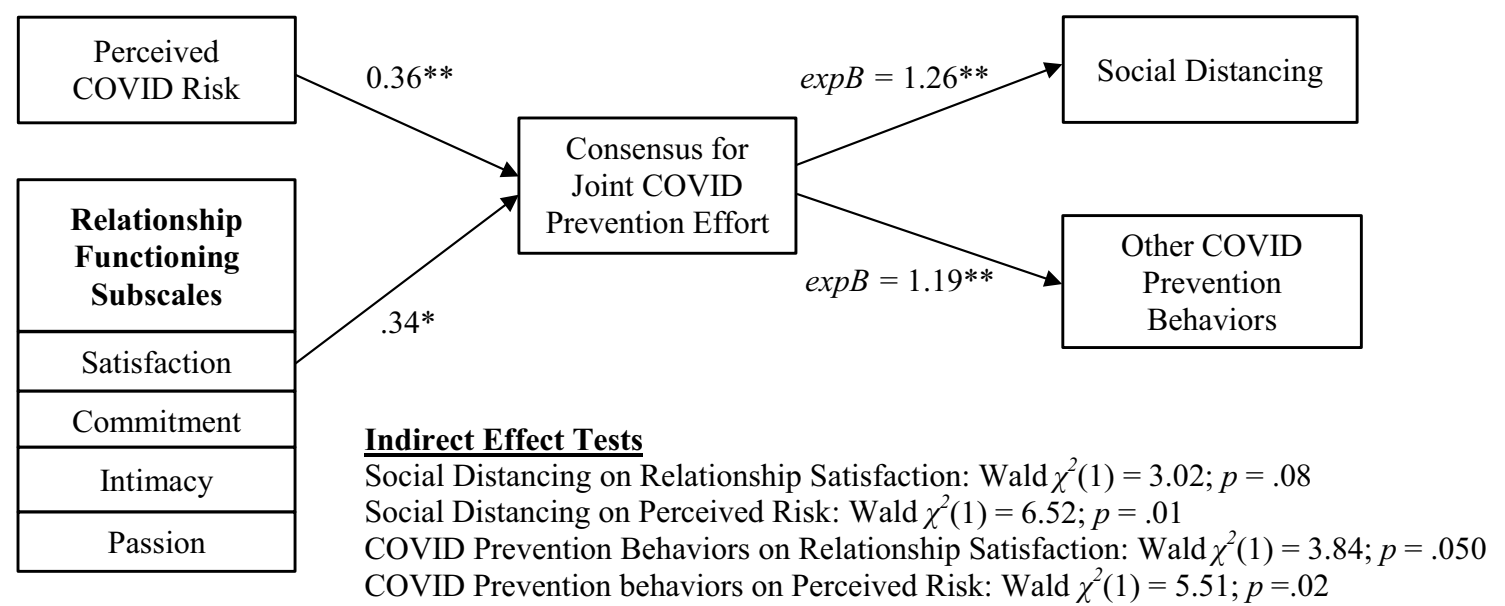

Fig. 1 Indirect effects on COVID-19 behavioral prevention methods via consensus for joint COVID-19 prevention effort 


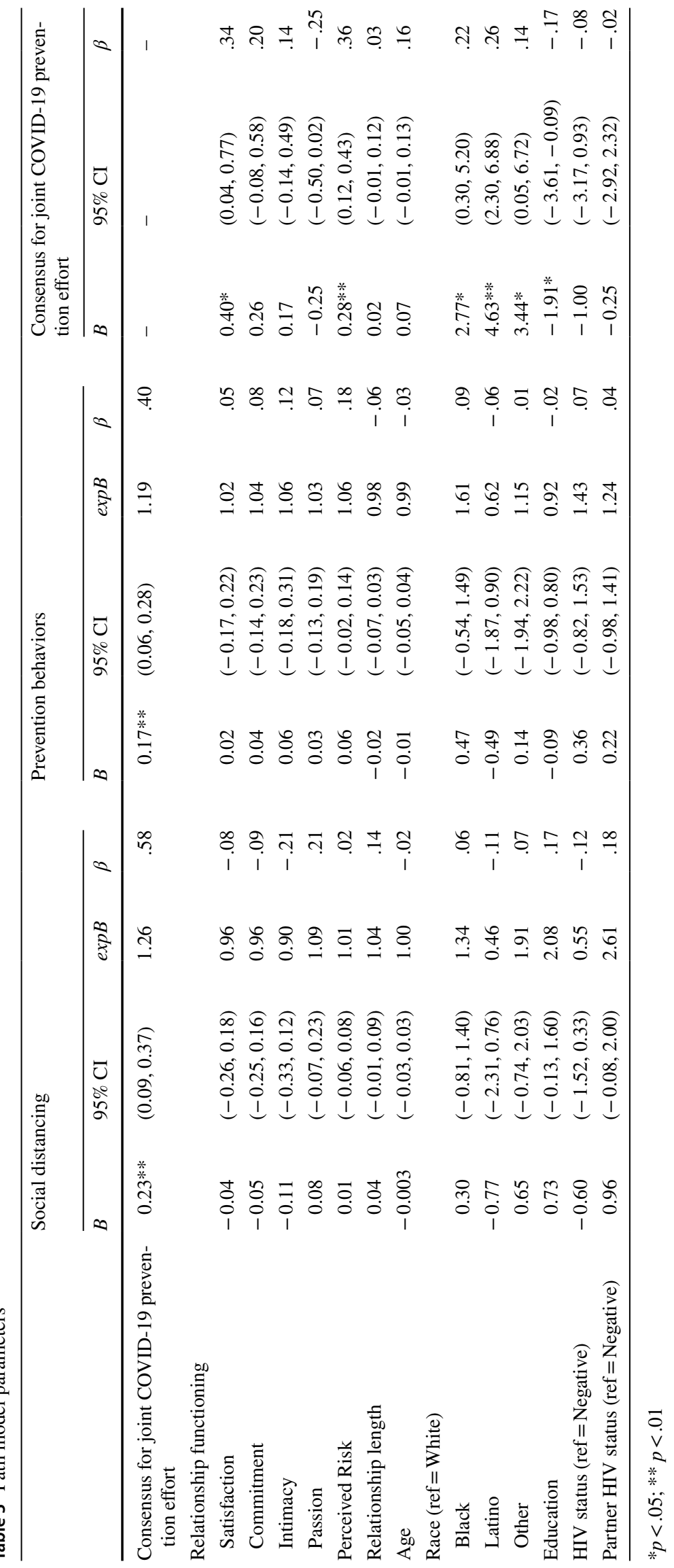




\section{Discussion}

The results of the current study largely conform to hypotheses grounded in interdependence theory (Kelley \& Thibaut, 1978; Rusbult \& Van Lange, 2003, 2008; Rusbult et al., 1991). They suggest that the concept of consensus or agreement about joint coping may be useful to understand the influence of relationship partners on COVID-19 prevention among SMM. Consensus for joint COVID-19 prevention effort-the extent to which partners were "on the same page" or had similar attitudes about COVID-19 prevention-was a central component in significant indirect paths linking perceived risk with COVID-19 prevention behaviors. It was also a central component in the indirect path linking relationship satisfaction with COVID-19 prevention behaviors that was at the threshold for significance $(p=.05)$. Collectively, these findings underscore the potential utility of exploring the possibility that dyadic interventions to reduce HIV acquisition through the formation of agreements about joint prevention goals might serve as a starting point for interventions to reduce COVID-19 risk among SMM couples.

The measure of consensus for joint effort used in this study assessed the alignment of partners' perspectives on COVID19 prevention. Previous research on congruence-the use of similar coping strategies by partners-has found it to be associated with relationship quality and individual health outcomes in heterosexual couples (Badr, 2004). Consistent with this, the findings presented here suggest that perceptions of congruent attitudes about COVID-19 prevention were predicted by relationship satisfaction and, in turn, were associated with engagement in COVID-19 prevention behavior.

The salience of congruence is illustrated by the fact that consensus for joint prevention effort predicted prevention behaviors (social distancing and other prevention strategies) above and beyond relationship functioning subscales, which were not significant direct predictors of either prevention outcome in multivariable models. A number of studies guided by Interdependence Theory have also illustrated the importance of goal congruence among SMM. In the context of health behavior, agreement on the outcomes of health-enhancing behaviors is believed to be an important predictor in joint engagement in health behaviors among couples (Lewis et al., 2006). Similarly, in an online convenience sample of MSM, an agreement between partners on sexual health outcomes resulted in greater engagement in risk reduction behaviors (Salazar et al., 2013). The authors argued that dyadic interventions aimed at reducing HIV transmission risk need to facilitate attitude alignment among couples as an effective intervention strategy (Salazar et al., 2013).

Although the commitment and intimacy subscales of relationship functioning had significant bivariate associations with consensus about joint COVID-19 prevention effort in a manner consistent with Interdependence Theory, they did not remain significant in multivariable models. This is probably best understood as an indication of the relative salience of relationship satisfaction in this sample, consistent with previous research investigating the association between relationship functioning and joint coping more broadly. A moderate to strong association between relationship satisfaction and dyadic coping was identified in a meta-analytic review of 39 studies (Falconier \& Kuhn, 2019). This association has also been identified among heterosexual couples coping with health concerns such as cancer, infertility, and organ transplants (e.g., Badr et al., 2010; Molgora et al., 2019; Tkachenko et al., 2019; Van Schoors et al., 2019) and similar findings have been identified among partnered SMM in the realm of HIVrelated health (Gamarel \& Revenson, 2015; Martins et al., 2020) and non-HIV health concerns (Randall et al., 2017a, 2017b; Rosenthal et al., 2019; Umberson et al., 2018).

Compared to relationship satisfaction, the standardized regression coefficients of commitment and intimacy in our multivariable models were approximately half the magnitude (or less). Although not as robust as findings related to relationship satisfaction, previous research has identified a significant positive association between dyadic coping and relationship commitment (Landis et al., 2014) as well as intimacy (Belcher et al., 2011; Manne \& Badr, 2010; Manne et al., 2010; Pagani et al., 2019; Traa et al., 2015) among heterosexual married couples. Similar associations between dyadic coping and relationship functioning have been identified among SMM couples (Feinstein et al., 2018; Meuwly et al., 2013; Rostosky \& Riggle, 2017; Song et al., 2020). Given the modest size of this sample, these findings are best viewed as indicating merit in the continued examination of relationship functioning generally as a determinant of COVID-19 prevention and related health outcomes and not as evidence that commitment or intimacy specifically are irrelevant.

Racial and ethnic minority populations generally and SMM of color specifically have endured disproportionately higher rates of SARS-CoV-2 infection and COVID-19-related hospitalizations (Hooper et al., 2020; Poulson et al., 2020). In this study, SMM of color had significantly higher levels of consensus about joint COVID-19 prevention effort compared to the majority of White participants. It is plausible that a constellation of factors including social and structural determinants of health (i.e., medical mistrust, healthcare access, and quality; Cahill et al., 2017; Eaton et al., 2015; Kimball et al., 2020) and social and structural racism (Díaz et al., 2001; Ibañez et al., 2012; Souleymanov et al., 2020) may increase the degree to which SMM of color rely on their partners as a source of information and support in coping with health risk. 
COVID-19 risk perception was not associated with prevention behavior in bivariate or multivariable analyses. This may arise at least partly because many prevention behaviors are determined in part by factors outside individual control. For example, essential workers may be less able to implement behavioral prevention strategies due to work demands (Robles et al., 2021). While not directly associated with prevention behavior, individual risk perception was a significant predictor of consensus about joint COVID-19 prevention effort. The fact that individual risk perception was associated with prevention behavior through agreement about joint effort is consistent with the premise that couple-level factors such as congruence exert a powerful influence on individual health behavior.

Risk perception has received relatively limited attention in Interdependence Theory research to date. It is plausible that risk perception provides some motivation for joint coping processes. If partners perceive COVID-19 to pose a substantial health risk, they may also experience an urgency around prevention. In contrast, partners who perceive COVID-19 as posing limited risk might not work together to prevent it simply because they do not believe the effort is warranted. These findings suggest that future research should explore the role of risk perceptions as a source of motivation for joint coping. To the extent that it serves as a catalyst or activating factor triggering joint coping processes, failure to account for its role may confound conclusions about associations between relationship functioning and joint coping.

A number of dyadic interventions have been developed to reduce HIV risk among SMM in relationships (Gamarel et al., 2019; Newcomb et al., 2017; Starks et al., 2019a, 2019c; Stephenson et al., 2016, 2017). All these interventions in some way are predicated on the premise that the formation of joint goals-a shared plan to promote health behavior-leads to improved health outcomes. Recently, Eaton and Kalichman (2020) have suggested that HIV prevention interventions may be leveraged to promote COVID-19 prevention. Findings from our study provide initial evidence that interventions that seek to use joint goal formation as a mechanism of intervention might be effective at promoting COVID-19 prevention behaviors among partnered SMM.

The findings must be understood in light of several limitations. First, although the relevance of joint coping has been illustrated in couples that are diverse with respect to gender composition (Falconier \& Kuhn, 2019), the prevention of a communicable virus may have unique salience for SMM couples and other populations at elevated risk of acquiring HIV, such as transwomen. Many SMM are socialized to consider health risk behavior and risk reduction in the context of their romantic relationships. For example, discussions between partners about sexual agreements and HIV prevention practices are commonly reported (e.g., Darbes et al., 2014; Hoff et al., 2009, 2010; Stephenson et al., 2015b). Therefore, SMM couples may be more accustomed to enacting health risk reduction strategies in the context of a dyad or romantic partnership compared to groups with lower HIV risk.

Second, generalizability was limited by convenience sampling from online dating and social networking spaces. Results may not generalize to SMM couples who are not engaged in such venues. Third, the data are cross-sectional. Therefore, interpretation of indirect effects is limited only to the quantification of shared variance and should not be interpreted as evidence of causal associations. Fourth, the study utilized a number of adapted measures or measures normed on heterosexual samples. Fifth, individuals who had contracted SARS-CoV-2 were excluded because they did not provide data on COVID-19 risk perception. At the time of data collection, the potential for re-infection was unclear. Finally, and possibly most important, we collected data about dyadic behavior from only one member of the couple. It was therefore not possible to examine partner influences on each other and on the couple's behavior.

Despite these limitations, the present study provides initial evidence that relationship functioning is associated with the individual-level implementation of social distancing and other COVID-19 prevention behaviors. Consistent with previous research on interdependence theory and dyadic coping, perceptions of congruence in partners' attitudes toward COVID19 prevention were predicted by relationship satisfaction and associated with individual COVID-19 prevention behavior. The findings support the premise that dyadic interventions to prevent HIV acquisition might be leveraged as a starting point for COVID-19 prevention with SMM in relationships.

Acknowledgements Analyses of these data were supported in part by a grant from the National Institute on Drug Abuse (R01 DA045613, PI: Starks). The authors thank the participants for their time and engagement. The authors also thank the study team, including S. Scott Jones, Ruben Jimenez, Trinae Adebayo, Brett Millar, Cynthia Cabral, Christine Cowles, Michael Suarez, Kory Kyre, Sugandha Gupta, and Daniel Sauermilch.

\section{Declarations}

Conflict of Interest The authors declare that they have no conflict of interest.

Ethical Approval All procedures performed in studies involving human participants were in accordance with the ethical standards of the institution at which the studies were conducted.

Informed Consent Informed consent was obtained from all individual participants included in the study.

\section{References}

Agnew, C. R., Van Lange, P. A. M., Rusbult, C. E., \& Langston, C. A. (1998). Cognitive interdependence: Commitment and the mental 
representation of close relationships. Journal of Personality and Social Psychology, 74(4), 939-954.

Albuquerque, G. A., de Lima Garcia, C., da Silva Quirino, G., Alves, M. J. H., Belém, J. M., dos Santos Figueiredo, F. W., da Silva Paiva, L., do Nascimento, V. B., da Silva Maciel, É., \& Valenti, V. E. (2016). Access to health services by lesbian, gay, bisexual, and transgender persons: Systematic literature review. BMC International Health and Human Rights, 16(1), 1-10. https://doi.org/10. 1186/s12914-015-0072-9.

Badr, H. (2004). Coping in marital dyads: A contextual perspective on the role of gender and health. Personal Relationships, 11(2), 197-211.

Badr, H., Carmack, C. L., Kashy, D. A., \& Cristofanilli, M. (2010). Dyadic coping in metastatic breast cancer. Health Psychology, 29(2), 169-180.

Banerjee, D., \& Nair, V. S. (2020). "The untold side of COVID-19": Struggle and perspectives of the sexual minorities. Journal of Psychosexual Health, 2(2), 113-120.

Belcher, A. J., Laurenceau, J. P., Graber, E. C., Cohen, L. H., Dasch, K. B., \& Siegel, S. D. (2011). Daily support in couples coping with early stage breast cancer: Maintaining intimacy during adversity. Health Psychology, 30(6), 665-673.

Berg, C. A., \& Upchurch, R. (2007). A developmental-contextual model of couples coping with chronic illness across the adult life span. Psychological Bulletin, 133(6), 920-954.

Blosnich, J. R., Hanmer, J., Yu, L., Matthews, D. D., \& Kavalieratos, D. (2016). Health care use, health behaviors, and medical conditions among individuals in same-sex and opposite-sex partnerships: A cross-sectional observational analysis of the Medical Expenditures Panel Survey (MEPS), 2003-2011. Medical Care, 54(6), 547-554.

Bodenmann, G., Meuwly, N., \& Kayser, K. (2011). Two conceptualizations of dyadic coping and their potential for predicting relationship quality and individual well-being. European Psychologist, 16(4), 255-266.

Bosco, S. C., Pawson, M., Parsons, J. T., \& Starks, T. J. (2021). Biomedical HIV prevention among gay male couples: A qualitative study of motivations and concerns. Journal of Homosexuality, $68,1353-1370$.

Brennan, D. J., Card, K. G., Collict, D., Jollimore, J., \& Lachowsky, N. J. (2020). How might social distancing impact gay, bisexual, queer, trans and two-spirit men in Canada? AIDS and Behavior, 24, 2480-2482.

Caceres, B. A., Makarem, N., Hickey, K. T., \& Hughes, T. L. (2019). Cardiovascular disease disparities in sexual minority adults: An examination of the behavioral risk factor surveillance system (2014-2016). American Journal of Health Promotion, 33(4), $576-585$.

Cahill, S., Taylor, S. W., Elsesser, S. A., Mena, L., Hickson, D., \& Mayer, K. H. (2017). Stigma, medical mistrust, and perceived racism may affect PrEP awareness and uptake in black compared to white gay and bisexual men in Jackson, Mississippi and Boston, Massachusetts. AIDS Care, 29(11), 1351-1358.

Centers for Disease Control and Prevention. (2020). Social distancing: Keep a safe distance to slow the spread. https://www.cdc.gov/ coronavirus/2019-ncov/prevent-getting-sick/social-distancing. html

Charlton, B. M., Gordon, A. R., Reisner, S. L., Sarda, V., Samnaliev, M., \& Austin, S. B. (2018). Sexual orientation-related disparities in employment, health insurance, healthcare access and healthrelated quality of life: A cohort study of US male and female adolescents and young adults. British Medical Journal Open, 8(6), e020418.

Cohen, S. E., Vittinghoff, E., Bacon, O., Doblecki-Lewis, S., Postle, B. S., Feaster, D. J., Matheson, T., Trainor, N., Blue, R. W., \& Estrada, Y. (2015). High interest in pre-exposure prophylaxis among men who have sex with men at risk for HIV-infection: Baseline data from the US PrEP demonstration project. Journal of Acquired Immune Deficiency Syndromes, 68(4), 439-448.

Cooper, A. N., Totenhagen, C. J., Curran, M. A., Randall, A. K., \& Smith, N. E. (2017). Daily relationship quality in same-sex couples: Attachment and sacrifice motives. Evolutionary Behavioral Sciences, 11(2), 146-160.

Courtemanche, C., Garuccio, J., Le, A., Pinkston, J., \& Yelowitz, A. (2020). Strong social distancing measures in the United States reduced the COVID-19 growth rate. Health Affairs, 39(7), $1237-1246$.

Curry, C. W., Felt, D., Beach, L. B., Ruprecht, M. M., Wang, X., \& Phillips, G. L. (2020). Lifetime asthma prevalence and correlates among US youths by sexual identity and race/ethnicity, 20092017. American Journal of Public Health, 110(7), 1076-1083.

Darbes, L. A., Chakravarty, D., Neilands, T. B., Beougher, S. C., \& Hoff, C. C. (2014). Sexual risk for HIV among gay male couples: A longitudinal study of the impact of relationship dynamics. Archives of Sexual Behavior, 43(1), 47-60.

Davidovich, U., de Wit, J. B. F., \& Stroebe, W. (2006). Relationship characteristics and risk of HIV infection: Rusbult's investment model and sexual risk behavior of gay men in steady relationships. Journal of Applied Social Psychology, 36(1), 22-40.

Díaz, R. M., Ayala, G., Bein, E., Henne, J., \& Marin, B. V. (2001). The impact of homophobia, poverty, and racism on the mental health of gay and bisexual Latino men: Findings from 3 US cities. American Journal of Public Health, 91(6), 927-932.

Eaton, L. A., Driffin, D. D., Kegler, C., Smith, H., Conway-Washington, C., White, D., \& Cherry, C. (2015). The role of stigma and medical mistrust in the routine health care engagement of black men who have sex with men. American Journal of Public Health, 105(2), e75-e82.

Eaton, L. A., \& Kalichman, S. C. (2020). Social and behavioral health responses to COVID-19: Lessons learned from four decades of an HIV pandemic. Journal of Behavioral Medicine, 43, 341-345. https://doi.org/10.1007/s10865-020-00157-y

Eikenberry, S. E., Mancuso, M., Iboi, E., Phan, T., Eikenberry, K., Kuang, Y., Kostelich, E., \& Gumel, A. B. (2020). To mask or not to mask: Modeling the potential for face mask use by the general public to curtail the COVID-19 pandemic. Infectious Disease Modelling, 5, 293-308.

Falconier, M. K., \& Kuhn, R. (2019). Dyadic coping in couples: A conceptual integration and a review of the empirical literature. Frontiers in Psychology, 10, 571. https://doi.org/10.3389/fpsyg. 2019.00571

Feinstein, B. A., McConnell, E., Dyar, C., Mustanski, B., \& Newcomb, M. E. (2018). Minority stress and relationship functioning among young male same-sex couples: An examination of actor-partner interdependence models. Journal of Consulting and Clinical Psychology, 86(5), 416-426.

Fisher, C. B., Fried, A. L., Macapagal, K., \& Mustanski, B. (2018). Patient-provider communication barriers and facilitators to HIV and STI preventive services for adolescent MSM. AIDS and Behavior, 22(10), 3417-3428.

Fletcher, G. J. O., Simpson, J. A., \& Thomas, G. (2000). The measurement of perceived relationship quality components: A confirmatory factor analytic approach. Personality and Social Psychology Bulletin, 26(3), 340-354.

Gamarel, K. E., Darbes, L. A., Hightow-Weidman, L., Sullivan, P., \& Stephenson, R. (2019). The development and testing of a relationship skills intervention to improve HIV prevention uptake among young gay, bisexual, and other men who have sex with men and their primary partners (We Prevent): Protocol for a randomized controlled trial. JMIR Research Protocols, 8(1), e10370. https:// doi.org/10.2196/10370 
Gamarel, K. E., \& Golub, S. A. (2019). Closeness discrepancies and intimacy interference: Motivations for HIV prevention behavior in primary romantic relationships. Personality and Social Psychology Bulletin, 45(2), 270-283.

Gamarel, K. E., \& Revenson, T. A. (2015). Dyadic adaptation to chronic illness: The importance of considering context in understanding couples' resilience. In K. Skerrett \& K. Fergus (Eds.), Couple resilience: Emerging perspectives (pp. 83-105). Springer.

Gamarel, K. E., Starks, T. J., Dilworth, S. E., Neilands, T. B., Taylor, J. M., \& Johnson, M. O. (2014). Personal or relational? Examining sexual health in the context of HIV serodiscordant same-sex male couples. AIDS and Behavior, 18(1), 171-179.

Godfrey, L. M., Whitton, S. W., Dyar, C., Newcomb, M. E., \& Mustanski, B. (2021). Sexual agreements among young sexual and gender minorities assigned male at birth: Associations with relationship quality and break-up. Archives of Sexual Behavior, 50, 1035-1045.

Goldenberg, T., Clarke, D., \& Stephenson, R. (2013). "Working together to reach a goal": MSM's perceptions for dyadic HIV care for same-sex male couples. Journal of Acquired Immune Deficiency Syndromes, 64(1), S52-S61.

Goldenberg, T., Finneran, C., Andes, K. L., \& Stephenson, R. (2015). "Sometimes people let love conquer them": How love, intimacy, and trust in relationships between men who have sex with men influence perceptions of sexual risk and sexual decision-making. Culture, Health \& Sexuality, 17(5), 607-622.

Grabbe, K., Bachanas, P., Moore, J., Rogers, M. F., \& Fenley, M. A. (2012). Couples HIV testing and counseling (CHTC) in health care facilities: Trainers Manual. The Centers for Disease Control. Retrieved June 15, 2021 from https://www.google.com/url?sa= $\mathrm{t} \& \mathrm{rct}=\mathrm{j} \& \mathrm{q}=\& \mathrm{esrc}=\mathrm{s} \&$ source $=$ web $\& \mathrm{~cd}=\& \mathrm{ved}=2 \mathrm{ahUKEwibj}$ saPwZrxAhUfRzABHclZBQMQFjAAegQICRAD\&url=https\% 3A\%2F\%2Fstacks.cdc.gov\%2Fview\%2Fcdc\%2F46952\%2Fcdc 46952_DS1.pdf\%3F\&usg=AOvVaw1p7HkXooOQsVh8Boaoi t7Q

Hoff, C. C., Beougher, S. C., Chakravarty, D., Darbes, L. A., \& Neilands, T. B. (2010). Relationship characteristics and motivations behind agreements among gay male couples: Differences by agreement type and couple serostatus. AIDS Care, 22(7), 827-835.

Hoff, C. C., Chakravarty, D., Beougher, S. C., Darbes, L. A., Dadasovich, R., \& Neilands, T. B. (2009). Serostatus differences and agreements about sex with outside partners among gay male couples. AIDS Education \& Prevention, 21(1), 25-38.

Hooper, M. W., Nápoles, A. M., \& Pérez-Stable, E. J. (2020). COVID19 and racial/ethnic disparities. Journal of the American Medical Association, 323(24), 2466-2467.

Ibañez, G. E., Marin, B. V. O., Flores, S. A., Millett, G., \& Diaz, R. M. (2012). General and gay-related racism experienced by Latino gay men. Cultural Diversity and Ethnic Minority Psychology, $15,215-222$.

Johnson, D. J., \& Rusbult, C. E. (1989). Resisting temptation: Devaluation of alternative partners as a means of maintaining commitment in close relationships. Journal of Personality and Social Psychology, 57(6), 967-980.

Johnson, M. O., Dilworth, S. E., Taylor, J. M., Darbes, L. A., Comfort, M. L., \& Neilands, T. B. (2012). Primary relationships, HIV treatment adherence, and virologic control. AIDS and Behavior, 16(6), $1511-1521$.

Kelley, H. H., \& Thibaut, J. W. (1978). Interpersonal relations: A theory of interdependence. Wiley.

Kimball, D., Rivera, D., Gonzales, M., \& Blashill, A. J. (2020). Medical mistrust and the PrEP cascade among Latino sexual minority men. AIDS and Behavior, 24, 3456-3461.

Landis, M., Bodenmann, G., Bradbury, T. N., Brandstätter, V., Peter-Wight, M., Backes, S., Sutter-Stickel, D., \& Nussbeck, F. W. (2014). Commitment and dyadic coping in long-term relationships. GeroPsych: The Journal of Gerontopsychology and Geriatric Psychiatry, 27(4), 139-149.

Lewis, M. A., McBride, C. M., Pollak, K. I., Puleo, E., Butterfield, R. M., \& Emmons, K. M. (2006). Understanding health behavior change among couples: An interdependence and communal coping approach. Social Science \& Medicine, 62(6), 1369-1380.

Lewnard, J. A., \& Lo, N. C. (2020). Scientific and ethical basis for social-distancing interventions against COVID-19. The Lancet Infectious Diseases, 20(6), 631-633.

Macapagal, K., Moskowitz, D. A., Li, D. H., Carrión, A., Bettin, E., Fisher, C. B., \& Mustanski, B. (2018). Hookup app use, sexual behavior, and sexual health among adolescent men who have sex with men in the United States. Journal of Adolescent Health, 62(6), 708-715.

Manne, S., \& Badr, H. (2010). Intimacy processes and psychological distress among couples coping with head and neck or lung cancers. Psycho-Oncology, 19(9), 941-954.

Manne, S., Badr, H., Zaider, T., Nelson, C., \& Kissane, D. (2010). Cancer-related communication, relationship intimacy, and psychological distress among couples coping with localized prostate cancer. Journal of Cancer Survivorship, 4(1), 74-85.

Martins, A., Canavarro, M. C., \& Pereira, M. (2020). The relationship between dyadic coping and dyadic adjustment among HIV-serodiscordant couples. AIDS Care, 33(4), 413-422.

Meuwly, N., Feinstein, B. A., Davila, J., Nuñez, D. G., \& Bodenmann, G. (2013). Relationship quality among Swiss women in oppositesex versus same-sex romantic relationships. Swiss Journal of Psychology, 72(4), 229-233.

Millar, B. M., Adebayo, T., Dellucci, T. V., Behar, E., \& Starks, T. J. (2020). Keeps me awake at night: The potential of the COVID-19 pandemic to affect sleep quality among sexual minority men in the U.S.A. Psychology of Sexual Orientation and Gender Diversity. https://doi.org/10.1037/sgd0000441

Mitchell, J., Brown, S., Pennington, J., Wu, Y. Y., \& Konda, K. A. (2019). Same-sex male couples' characteristics and attitudes towards different HIV prevention services by dyad HIV serostatus in Lima Peru. International Journal of Sexual Health, 31(4), 375-383. https://doi.org/10.1080/19317611.2019.1662532

Mitchell, J. W., \& Petroll, A. E. (2013). Factors associated with men in HIV-negative gay couples who practiced UAI within and outside of their relationship. AIDS and Behavior, 17(4), 1329-1337. https://doi.org/10.1007/s10461-012-0255-5

Molgora, S., Fenaroli, V., Acquati, C., De Donno, A., Baldini, M. P., \& Saita, E. (2019). Examining the role of dyadic coping on the marital adjustment of couples undergoing assisted reproductive technology (ART). Frontiers in Psychology, 10, 415. https://doi. org/10.3389/fpsyg.2019.00415.

Muthén, L. K., \& Muthén, B. O. (2018). Mplus users guide and Mplus version 8.2 . http://www.statmodel.com/index.shtml

Muusses, L. D., Kerkhof, P., \& Finkenauer, C. (2015). Internet pornography and relationship quality: A longitudinal study of within and between partner effects of adjustment, sexual satisfaction and sexually explicit internet material among newly-weds. Computers in Human Behavior, 45, 77-84.

Napper, L. E., Fisher, D. G., \& Reynolds, G. L. (2012). Development of the perceived risk of HIV scale. AIDS and Behavior, 16(4), 1075-1083.

Newcomb, M. E., Macapagal, K. R., Feinstein, B. A., Bettin, E., Swann, G., \& Whitton, S. W. (2017). Integrating HIV prevention and relationship education for young same-sex male couples: A pilot trial for the 2GETHER intervention. AIDS and Behavior, 21(8), 2464-2478.

Nguyen, K. H., Trivedi, A. N., \& Shireman, T. I. (2018). Lesbian, gay, and bisexual adults report continued problems affording care despite coverage gains. Health Affairs, 37(8), 1306-1312. 
Operario, D., Gamarel, K. E., Grin, B. M., Lee, J. H., Kahler, C. W., Marshall, B. D. L., van den Berg, J. J., \& Zaller, N. D. (2015). Sexual minority health disparities in adult men and women in the United States: National Health and Nutrition Examination Survey, 2001-2010. American Journal of Public Health, 105(10), e27-e34.

Pagani, A. F., Donato, S., Parise, M., Bertoni, A., Iafrate, R., \& Schoebi, D. (2019). Explicit stress communication facilitates perceived responsiveness in dyadic coping. Frontiers in Psychology, 10, 401. https://doi.org/10.3389/fpsyg.2019.00401.

Poulson, M., Geary, A., Annesi, C., Allee, L., Kenzik, K., Sanchez, S., Tseng, J., \& Dechert, T. (2020). National disparities in COVID19 outcomes between Black and White Americans. Journal of the National Medical Association, 113(2), 125-132.

Randall, A. K., Tao, C., Totenhagen, C. J., Walsh, K. J., \& Cooper, A. N. (2017a). Associations between sexual orientation discrimination and depression among same-sex couples: Moderating effects of dyadic coping. Journal of Couple \& Relationship Therapy, 16(4), 325-345.

Randall, A. K., Totenhagen, C. J., Walsh, K. J., Adams, C., \& Tao, C. (2017b). Coping with workplace minority stress: Associations between dyadic coping and anxiety among women in same-sex relationships. Journal of Lesbian Studies, 21(1), 70-87.

Remien, R. H., Stirratt, M. J., Dolezal, C., Dognin, J. S., Wagner, G. J., Carballo-Diéguez, A., El-Bassel, N., \& Jung, T. M. (2005). Couple-focused support to improve HIV medication adherence: A randomized controlled trial. AIDS, 19(8), 807-814.

Robles, G., Sauermilch, D., \& Starks, T. J. (2021). Self-efficacy, social distancing, and essential worker status dynamics among SGM people. Annals of LGBTQ Public and Population Health, 1(4), 300-317.

Romanelli, M., \& Hudson, K. D. (2017). Individual and systemic barriers to health care: Perspectives of lesbian, gay, bisexual, and transgender adults. American Journal of Orthopsychiatry, 87(6), 714-728.

Rosenthal, L., Deosaran, A., Young, D. L., \& Starks, T. J. (2019). Relationship stigma and well-being among adults in interracial and same-sex relationships. Journal of Social and Personal Relationships, 36(11-12), 3408-3428.

Rostosky, S. S., \& Riggle, E. D. B. (2017). Same-sex couple relationship strengths: A review and synthesis of the empirical literature (2000-2016). Psychology of Sexual Orientation and Gender Diversity, 4(1), 1-13.

Rusbult, C. E. (1980). Commitment and satisfaction in romantic associations: A test of the investment model. Journal of Experimental Social Psychology, 16(2), 172-186.

Rusbult, C. E. (1983). A longitudinal test of the investment model: The development (and deterioration) of satisfaction and commitment in heterosexual involvements. Journal of Personality and Social Psychology, 45(1), 101-117.

Rusbult, C. E., Martz, J. M., \& Agnew, C. R. (1998). The Investment Model Scale: Measuring commitment level, satisfaction level, quality of alternatives, and investment size. Personal Relationships, 5(4), 357-391.

Rusbult, C. E., \& Van Lange, P. A. M. (2003). Interdependence, interaction, and relationships. Annual Review of Psychology, 54(1), 351-375.

Rusbult, C. E., \& Van Lange, P. A. M. (2008). Why we need interdependence theory. Social and Personality Psychology Compass, 2(5), 2049-2070.

Rusbult, C. E., Verette, J., Whitney, G. A., Slovik, L. F., \& Lipkus, I. (1991). Accommodation processes in close relationships: Theory and preliminary empirical evidence. Journal of Personality and Social Psychology, 60(1), 53-78.

Rusbult, C. E., Zembrodt, I. M., \& Gunn, L. K. (1983). Exit, voice, loyalty, and neglect: Responses to dissatisfaction in romantic involvements. Journal of Personality and Social Psychology, 43(6), 1230-1242.

Sabin, J. A., Riskind, R. G., \& Nosek, B. A. (2015). Health care providers' implicit and explicit attitudes toward lesbian women and gay men. American Journal of Public Health, 105(9), 1831-1841.

Salazar, L. F., Stephenson, R., Sullivan, P. S., \& Tarver, R. (2013). Development and validation of HIV-related dyadic measures for men who have sex with men. Journal of Sex Research, 50(2), 164-177.

Sanchez, T. H., Zlotorzynska, M., Rai, M., \& Baral, S. D. (2020). Characterizing the impact of COVID-19 on men who have sex with men across the United States in April, 2020. AIDS and Behavior, 24, 2024-2032.

Satorra, A., \& Bentler, P. M. (2001). A scaled difference chi-square test statistic for moment structure analysis. Psychometrika, 66(4), 507-514.

Silva, A., Saraiva, M., Albuquerque, P. B., \& Arantes, J. (2017). Relationship quality influences attitudes toward and perceptions of infidelity. Personal Relationships, 24(4), 718-728.

Song, C., Buysse, A., Zhang, W., Lu, C., Zhao, M., \& Dewaele, A. (2020). Coping with minority stress in romantic relationships among lesbian, gay and bisexual people. Current Psychology. https://doi.org/10.1007/s12144-020-01188-z

Souleymanov, R., Brennan, D. J., George, C., Utama, R., \& Ceranto, A. (2020). Experiences of racism, sexual objectification and alcohol use among gay and bisexual men of colour. Ethnicity \& Health, 25(4), 525-541.

Starks, T. J., Dellucci, T. V., Gupta, S., Robles, G., Stephenson, R., Sullivan, P., \& Parsons, J. T. (2019a). A pilot randomized trial of intervention components addressing drug use in Couples HIV Testing and Counseling (CHTC) with male couples. AIDS and Behavior, 23, 2407-2420. https://doi.org/10.1007/s10461-019-02455-2

Starks, T. J., Doyle, K. M., Shalhav, O., John, S. A., \& Parsons, J. T. (2019b). An examination of gay couples' motivations to use (or forego) pre-exposure prophylaxis expressed during couples HIV testing and counseling (CHTC) sessions. Prevention Science, 20(1), 157-167.

Starks, T. J., Ewing, S. W. F., Lovejoy, T., Gurung, S., Cain, D., Fan, C. A., Naar, S., \& Parsons, J. T. (2019c). Adolescent male couplesbased HIV testing intervention (We Test): Protocol for a Type 1, hybrid implementation-effectiveness trial. JMIR Research Protocols, 8(6), e11186. https://doi.org/10.2196/11186

Starks, T. J., Gamarel, K. E., \& Johnson, M. O. (2014). Relationship characteristics and HIV transmission risk in same-sex male couples in HIV sero-discordant relationships. Archives of Sexual Behavior, 43(1), 139-147.

Starks, T. J., Jones, S. S., Sauermilch, D., Benedict, M., Adebayo, T., Cain, D., \& Simpson, K. N. (2020a). Evaluating the impact of COVID-19: A cohort comparison study of drug use and risky sexual behavior among sexual minority men in the U.S.A. Drug and Alcohol Dependence. https://doi.org/10.1016/j.drugalcdep. 2020.108260

Starks, T. J., Robles, G., Bosco, S. C., Doyle, K. M., \& Dellucci, T. V. (2019d). Relationship functioning and substance use in samesex male couples. Drug and Alcohol Dependence, 201, 101-108.

Starks, T. J., Robles, G., Doyle, K. M., Pawson, M., Bertone, P. B., Millar, B. M., \& Ingersoll, K. S. (2020b). Motivational Interviewing with male couples to reduce substance use and HIV risk: Manifestations of partner discord and strategies for facilitating dyadic functioning. Psychotherapy, 57(1), 58-67. https://doi.org/ $10.1037 /$ pst0000278

Stephenson, R., Freeland, R., Sullivan, S. P., Riley, E., Johnson, B. A., Mitchell, J., McFarland, D., \& Sullivan, P. S. (2017). Home-based HIV testing and counseling for male couples (Project Nexus): A 
protocol for a randomized controlled trial. JMIR Research Protocols, 6(5), e101. https://doi.org/10.2196/resprot.7341

Stephenson, R., Grabbe, K. L., Sidibe, T., McWilliams, A., \& Sullivan, P. S. (2016). Technical assistance needs for successful implementation of Couples HIV testing and Counseling (CHTC) intervention for male couples at US HIV testing sites. AIDS and Behavior, 20,841-847.

Stephenson, R., Vwalika, B., Greenberg, L. S., Ahmed, Y., Vwalika, C., Chomba, E., Kilembe, W., Tichacek, A., \& Allen, S. (2011). A randomized controlled trial to promote long-term contraceptive use among HIV-serodiscordant and concordant positive couples in Zambia. Journal of Women's Health, 20(4), 567-574.

Stephenson, R., White, D., Darbes, L., Hoff, C., \& Sullivan, P. (2015a). HIV testing behaviors and perceptions of risk of HIV infection among MSM with main partners. AIDS and Behavior, 19(3), 553-560.

Stephenson, R., White, D., \& Mitchell, J. W. (2015b). Sexual agreements and perception of HIV prevalence among an online sample of partnered men who have sex with men. Archives of Sexual Behavior, 44(7), 1813-1819.

Sullivan, P. S., White, D., Rosenberg, E. S., Barnes, J., Jones, J., Dasgupta, S., O’Hara, B., Scales, L., Salazar, L. F., Wingood, G., DiClemente, R., Wall, K. M., Hoff, C. C., Gratzer, B., Allen, S., \& Stephenson, R. (2014). Safety and acceptability of couples HIV testing and counseling for US men who have sex with men: A randomized prevention study. Journal of the International Association of Providers of AIDS Care, 13(2), 135-144.

Thunström, L., Newbold, S. C., Finnoff, D., Ashworth, M., \& Shogren, J. F. (2020). The benefits and costs of using social distancing to flatten the curve for COVID-19. Journal of Benefit-Cost Analysis, 11(2), 179-195.

Tkachenko, D., Franke, L., Peters, L., Schiffer, M., \& Zimmermann, T. (2019). Dyadic coping of kidney transplant recipients and their partners: Sex and role differences. Frontiers in Psychology, 10, 397. https://doi.org/10.3389/fpsyg.2019.00397.

Torres, T. S., Hoagland, B., Bezerra, D. R. B., Garner, A., Jalil, E. M., Coelho, L. E., Benedetti, M., Pimenta, C., Grinsztejn, B., \&
Veloso, V. G. (2020). Impact of COVID-19 pandemic on sexual minority populations in Brazil: An analysis of social/racial disparities in maintaining social distancing and a description of sexual behavior. AIDS and Behavior, 25, 73-84.

Traa, M. J., De Vries, J., Bodenmann, G., \& Den Oudsten, B. L. (2015). Dyadic coping and relationship functioning in couples coping with cancer: A systematic review. British Journal of Health Psychology, 20(1), 85-114.

Umberson, D., Donnelly, R., \& Pollitt, A. M. (2018). Marriage, social control, and health behavior: A dyadic analysis of same-sex and different-sex couples. Journal of Health and Social Behavior, 59(3), 429-446.

Van Schoors, M., Loeys, T., Goubert, L., Berghmans, G., Ooms, B., Lemiere, J., Norga, K., \& Verhofstadt, L. L. (2019). Couples dealing with pediatric blood cancer: A study on the role of dyadic coping. Frontiers in Psychology, 10, 402. https://doi.org/10.3389/ fpsyg.2019.00402.

Veldhuis, C. B., Bruzzese, J. M., Hughes, T. L., \& George, M. (2019). Asthma status and risks among lesbian, gay, and bisexual adults in the United States: A scoping review. Annals of Allergy, Asthma \& Immunology, 122(5), 535-536.

Weston, R., \& Gore, P. A. (2006). A brief guide to structural equation modeling. The Counseling Psychologist, 34(5), 719-751.

Wu, E., El-Bassel, N., McVinney, L. D., Hess, L., Fopeano, M. V., Hwang, H. G., Charania, M., \& Mansergh, G. (2015). The association between substance use and intimate partner violence within Black male same-sex relationships. Journal of Interpersonal Violence, 30(5), 762-781.

Publisher's Note Springer Nature remains neutral with regard to jurisdictional claims in published maps and institutional affiliations. 